NEUROGENESIS

\section{New cells for old}

Primary neurons in the mammalian olfactory epithelium are continuously renewed, even during adulthood. According to Bauer et al., writing in The Journal of Neuroscience, leukaemia inhibitory factor (LIF) is the key to this neurogenesis, and is produced by dying neurons to stimulate their replacement.

LIF is a cytokine that is involved in regulating the differentiation of many cells, and is one of many factors that has been shown to stimulate the production of new olfactory neurons from their progenitors in vitro. Bauer et al. studied mice in which the olfactory bulbs had been ablated, leading to massive cell death in the olfactory epithelium followed by a wave of mitosis in the globose basal cells (which give rise to the olfactory neurons). When they examined the olfactory epithelium after this injury, they found that LIF was strongly induced. The LIF receptor is also upregulated during this period, but the authors found no other cytokines or growth factors that showed this pattern of

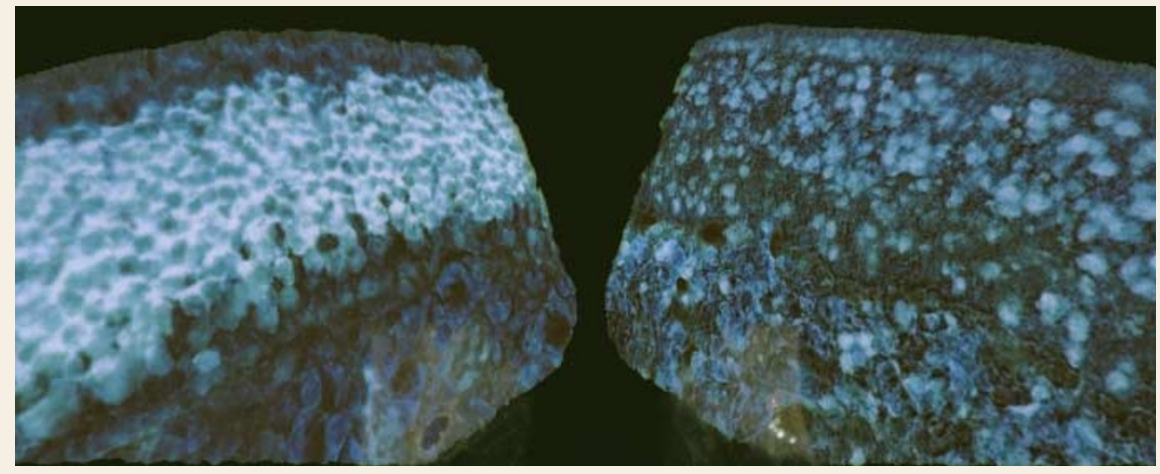

The left image is an in situ hybridization for olfactory marker protein, illustrating the location of the olfactory neurons in the epithelium. The right image is an in situ RT-PCR for leukaemia inhibitory factor (LIF), showing that olfactory neurons are positive for LIF mRNA after olfactory bulb ablation. Courtesy of P. H. Patterson, California Institute of Technology, Pasadena, California.

upregulation. LIF was induced in the neurons of the olfactory epithelium - the population of neurons that dies through apoptosis after olfactory bulb ablation.

To find out whether LIF is needed for the production of new olfactory neurons after injury, the authors used LIF knockout mice. In these mice, ablation of the olfactory bulb still led to apoptosis in the olfactory epithelium, but there was no increase in progenitor cell division. Finally, Bauer et al. induced overexpression of LIF in the olfactory epithelium of unlesioned mice using an adenoviral vector, and found that this caused increased neurogenesis in the apical olfactory epithelium.
Although there are a number of questions to be answered, the authors propose that LIF is released by dying olfactory neurons to stimulate their replacement through cell division. It will be interesting to see whether similar factors can be identified in other neuronal populations that have been reported to be replaced after apoptosis. If so, it could have important implications for brain repair.

Rachel Jones

(2) References and links ORIGINAL RESEARCH PAPER Bauer, S. et al. Leukemia inhibitory factor is a key signal for injury-induced neurogenesis in the adult mouse olfactory epithelium. J. Neurosci. 23, 1792-1803 (2003)

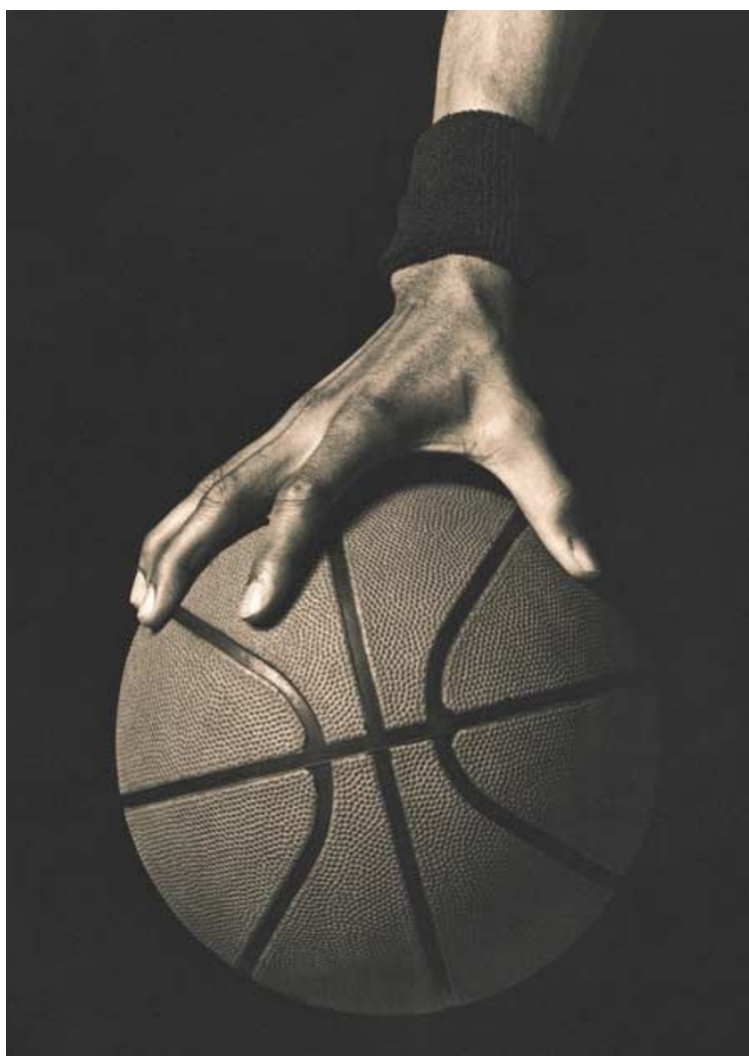

\title{
A good grasp on intraparietal areas
}

In monkeys, the anterior bank of the intraparietal sulcus (AIP) is involved in visually guided hand movements that are relevant to grasping objects. By contrast, its caudal bank (CIP) responds to three-dimensional surface orientation. On the basis of these findings, it has been proposed that CIP conveys spatial information to AIP, affecting motor output. Now, Shikata et al. report that the equivalent areas in the human brain show a similar division of labour, and suggest that these regions functionally interact during visually guided hand movements.

Using event-related functional imaging, the authors measured AIP and CIP activation in three different tasks: discrimination of surface orientation, imagination of the necessary movement to grasp a surface of a specific orientation, and execution of the actual movement. They found that, whereas CIP activation was similar in the three tasks, AIP activation was strongest when the movement was executed and weakest when the subject simply dis- criminated surface orientation. As Shikata et al. had previously shown that performance modulates CIP activity in the surface orientation task (its activation is stronger in successful trials), the authors now conclude that the functional dissociation between AIP and CIP originally observed in monkeys is also present in the human brain.

Although these findings are consistent with the idea that CIP might send information to AIP during visually guided hand movements, the rigorous testing of this idea will require the use of additional techniques such as transcranial magnetic stimulation during the performance of similar behavioural tasks.

(2) References and links Juan Carlos López original RESEARCH PAPERS Shikata, E. et Functional properties and interaction of the anterior and posterior intraparietal areas in humans. Eur. J. Neurosci. 17, 1105-1110 (2003) | Shikata, E. et al. Surface orientation discrimination activated caudal and anterior intraparietal sulcus in humans: an event-related fMRI study. J. Neurophysiol. 85, 1309-1313 (2003) 